\title{
Upgrade of the TAMU MDM-Focal Plane Detector with MicroMegas Technology
}

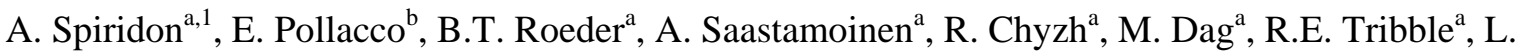 \\ Trache $^{\mathrm{c}}$, G. Pascovici ${ }^{\mathrm{c}}$, R. De Oliveira ${ }^{\mathrm{d}}$ \\ ${ }^{a}$ Cyclotron Institute, Texas A\&M University, College Station, TX-77843, USA \\ ${ }^{b}$ IRFU, CEA Saclay, Gif-sur-Yvette, France \\ ${ }^{c}$ National Institute of Physics and Nuclear Engineering, Bucharest-Magurele, RO-077125, Romania \\ ${ }^{d}$ CERN, Geneva, Switzerland
}

\begin{abstract}
A gridded ionization chamber used as a focal plane detector at the back of the TAMU-MDM spectrometer was modified to use MicroMegas technology for the purpose of improving energy resolution and particle identification. The upgraded system was tested in experimental conditions with several heavy-ion beams at $12 \mathrm{MeV} / \mathrm{u}$ and found to achieve resolutions between $3.2 \%$ and $4.8 \%$. This is a significant improvement over the previous performance of $10-15 \%$ obtained using the existing, conventional ionization chambers.
\end{abstract}

\section{Introduction}

X-ray bursts are the most frequent thermonuclear explosion occurring in the universe and represent one type of phenomena responsible for heavier element nucleosynthesis. For this reason and others, a number of powerful X-ray observatories have been used to take large amounts of data on these bursts. The interpretation of these observations, however, is problematic due to the lack of a complete understanding of the nuclear physics at the base of these phenomena [1]. Among the various processes occurring in X-ray bursts, the most important is the rp-process. It is dominated by $(p, \gamma),(\alpha, p)$ reactions and $\beta$-decays. Critical nuclear data is needed related to these processes such as: nuclear masses, $\beta$-decay rates and reaction rates. Of the three, reaction rates are the most difficult to determine with direct methods due to the fact that most of the nuclei involved in these reactions are unstable [2].

At Cyclotron Institute, Texas A\&M University, we have measured various proton capture reactions indirectly using the Asymptotic Normalization Coefficient (ANC) method [3] and experiments involving proton and neutron transfer reactions. These experiments were done primarily with the Multipole-Dipole-Multipole (MDM) spectrometer [4]. The Oxford focal plane detector sitting at the back was used to identify particles and measure their positions along the dispersive $\mathrm{x}$-direction. Using raytrace reconstruction we could determine the scattering angle at the target as a function of the angle of the particle path in the detector [5]. The reactions studied so far involved nuclei with $\mathrm{A} \leq 26$. For masses in that region, we found that we were having significant difficulties with the particle identification (PID) due to the insufficient resolution of both the $\Delta \mathrm{E}$ (energy lost in the gas) and $\mathrm{E}_{\text {res }}$ (residual energy, depozited in the stopping material) signals (see Figure 1). Specifically, we needed to analyze isotopes of masses A and A+1 separately but could not gate on each exclusively due to overlap.

\footnotetext{
1 *Corresponding author. Tel. +1 9798451411 E-mail address: aspiridon@comp.tamu.edu
} 


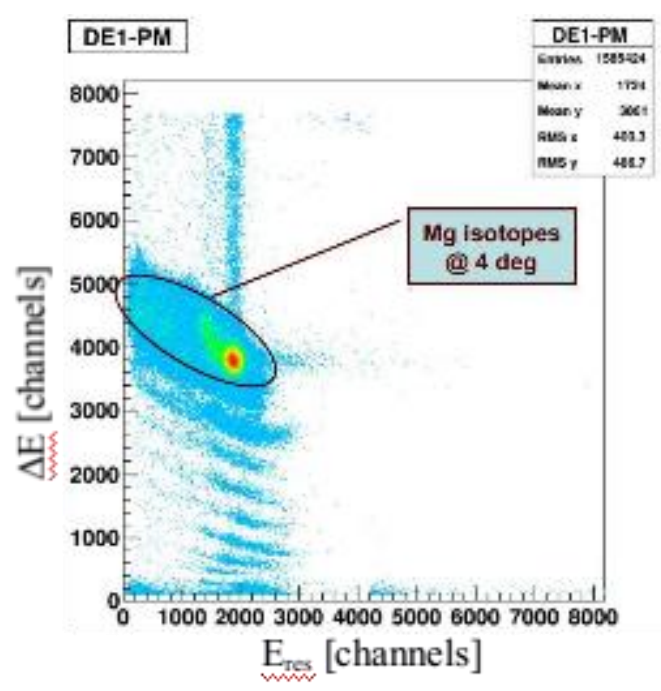

Figure 1. Standard 2D $\Delta \mathrm{E}_{-} \mathrm{E}_{\mathrm{res}}$ spectrum produced by the Oxford detector and used for PID

In order to improve $\Delta \mathrm{E}$, we decided to use a MicroMegas detector. This is a relatively new detector technology that we have successfully used in a different system, called Astrobox [6], which is built specifically for low noise and is used to detect very low-energy protons from beta-delayed proton emitters.

The MicroMegas detector operates as a two-stage parallel-plate avalanche chamber. It consists of a small amplification gap (50-300 $\mu \mathrm{m})$ and a much larger drift gap (on the order of $\mathrm{cm}$ ) separated by a thin electroformed micromesh. It has been shown to provide gains of up to $10^{5}$ [7].

In the course of testing and using the Astrobox, we observed that the MicroMegas also detected the incoming energetic heaver ions with good resolution for particle identification. In light of that, we decided that this technology was exactly what we needed to upgrade the Oxford focal plane detector.

\section{The TAMU-Focal Plane Detector}

The Oxford detector is a gridded ionization chamber with a plastic scintillator at the back. Figure 2 (top) shows a schematic side view of the detector and its components. Figure 2 (bottom) shows a photograph, similarly oriented for comparison. In both pictures, the beam of particles would come from the left. Four resistive wires working in avalanche conditions (AC) allow for position determination at four different depths in the detector and therefore permit raytrace reconstruction. Energy lost in the ionization gas, $\Delta \mathrm{E}$, is measured by the three anode plates at the top. Particles are stopped in the scintillator at the back, and the residual energy, $\mathrm{E}_{\text {res, }}$, is measured with two phototubes. A more detailed description of the detector, its components and its operation can be found in ref [8]. Currently, with only the first two plates connected to produce a signal we call $\Delta \mathrm{E} 1$, we obtain energy resolutions of $10-15 \%$, depending on voltage settings, gas pressure, particle $\mathrm{Z}$ and energy.

The third plate, $\Delta \mathrm{E} 2$, gave a signal with similar or poorer resolution and was never shown to improve particle identification. For this reason, we replaced the $\Delta E 2$ anode with a MicroMegas plate (type Bulk) of identical size, $14.6 \mathrm{~cm}$ by $42.6 \mathrm{~cm}$ (Figure 3, top). 


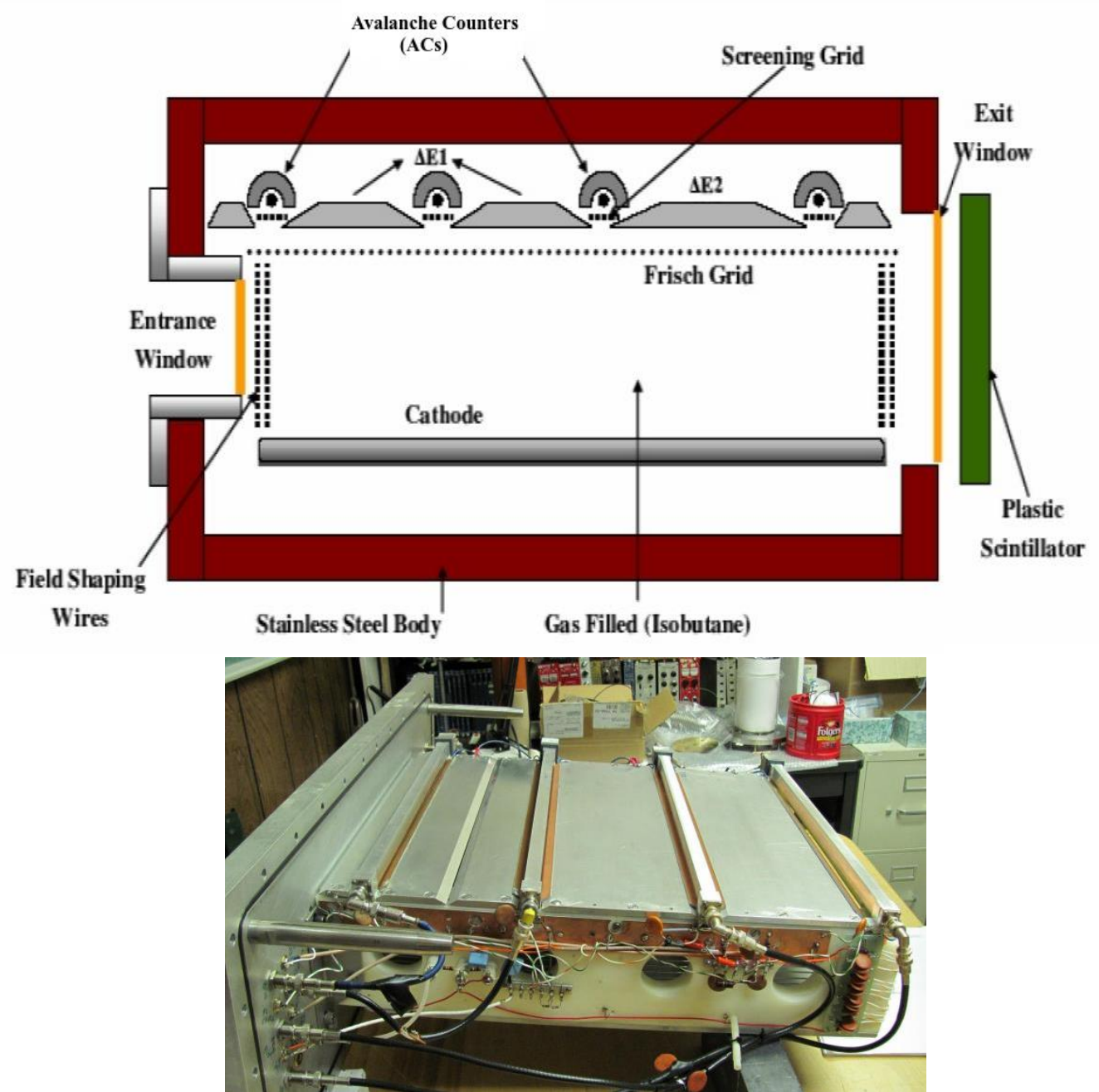

Figure 2. (Top) Schematic drawing of the Oxford. (Bottom) Photo showing the Oxford detector.

The detection area consisted of 28 pads, $3.25 \mathrm{~cm}$ by $4.4 \mathrm{~cm}$, each giving an individual $\Delta \mathrm{E}$ signal corresponding to the energy lost in the respective gas region. The mesh was made of nickel and created an amplification gap of $256 \mu \mathrm{m}$ with the anode. The drift region, between this mesh and the cathode was $12 \mathrm{~cm}$. The detector was filled with isobutane gas and was operated at different pressures, between 30 and 100 Torr.

The 28 signals were read out through two DSub-25 connectors (Figure 3, bottom), two rows of pads to each. Short flat cables routed the signals to two DSub-25 feedthroughs in the detector chamber. The electronics used to process them were: 2 Mesytec MPR-16 preamplifiers, 2 Mesytec MSCF-16 amplifiers and one VME-ADC module. The acquisition trigger was given by the coincidence between the phototube signals.

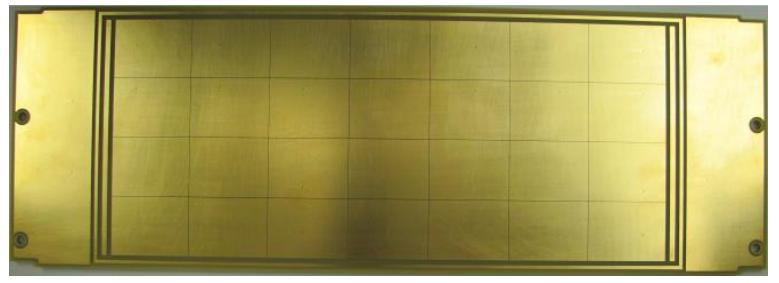




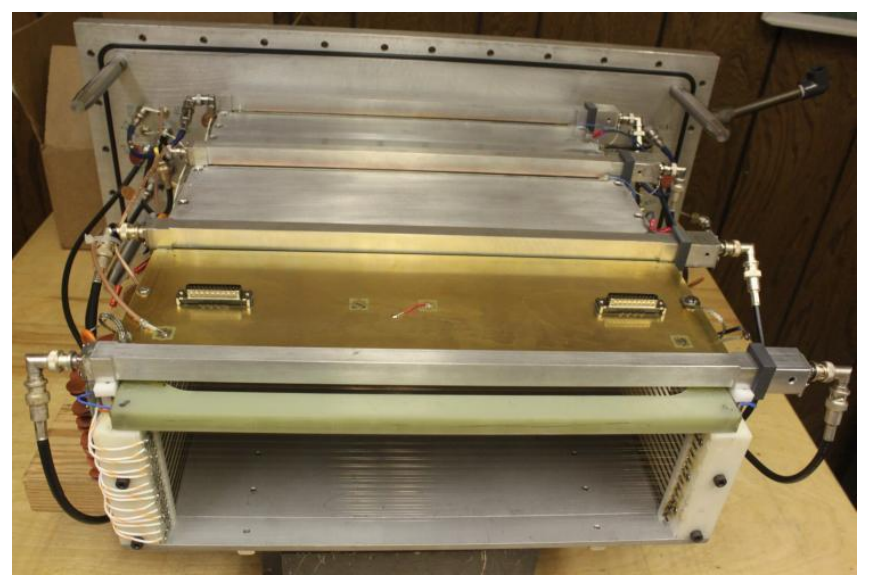

Figure 3. (Top)The Micromegas Anode. (Bottom) Oxford detector with the new Anode mounted.

\section{Results}

We tested the upgraded detector with 3 beams: ${ }^{16} \mathrm{O},{ }^{22} \mathrm{Ne}$ and ${ }^{28} \mathrm{Si}$. Each beam had an energy of $12 \mathrm{MeV} / \mathrm{u}$. We used mainly elastic scattering on ${ }^{197} \mathrm{Au}$. The heavy target allowed for a relatively pure beam (with some inelastic contributions). We also used ${ }^{27} \mathrm{Al}$ (only for ${ }^{16} \mathrm{O}$ ) and ${ }^{13} \mathrm{C}$ targets in order to produce a cocktail of nuclei and observe the resulting PID spectra.

The purpose of these test experiments was to determine the MicroMegas response to different settings. We looked at the detector behavior for different bias voltages, different gas pressures and particles of different $\mathrm{N}$ and $\mathrm{Z}$.

In studies done by other groups and also in our AstroBox tests, MicroMegas were only used with gases around atmospheric pressure. With the Oxford, we are limited by the entrance and exit windows so we had to start at 100 Torr, the maximum pressure we could safely have inside the detector without breaking the windows. However, this pressure is problematic at larger reaction angles where particles stop before triggering the data acquisition. In order to test the detector under the conditions of an ANC study we had to cover the lower pressure range as well, especially 30 Torr which is what we typically use for such studies.

For each beam, we focused on elastic scattering off the gold foil. The reaction products were collimated with a narrow slit before going into the spectrometer. This allowed us to test each column of 4 pads in the MicroMegas anode with the same beam energy distribution. As an example, Figure 4 shows the beam passing through the center of the Oxford detector, highlighting column 4 of the MicroMegas anode. The 4 position spectra detected by the ACs confirm this. Under these conditions, we observed the detector behavior for different bias voltages, gas pressures, and electronic gains.

An example of energy spectrum for an individual pad is shown in Figure 5. The label 'R1-C4' denotes that it corresponds to the center pad in the first row to meet the beam. The resolution of the peak is $\sim 5.5 \%$. For all three test beams, we obtained energy resolutions for the individual pads in the range of 5.5-6.5\% for 100 Torr and 85 Torr, $5.5-6 \%$ for 70 Torr, $6.5-7 \%$ for 50 Torr, and $8-9 \%$ for 30 Torr. Averaging the energy over all the pads gave us significantly better resolution (Figure 6).

Last but not least, we looked at a cocktail of reaction products using the ${ }^{27} \mathrm{Al}$ and ${ }^{13} \mathrm{C}$ targets for different MDM angles and compared the PID spectra we obtained with the spectra that were obtained before the modification. Figure 7 shows one such comparison for ${ }^{22} \mathrm{Ne}$ at 5 degrees and 
30 Torr. It can be easily seen that the better resolution of the MicroMegas translates into improved separation between the various particles detected.

The $\Delta \mathrm{E} 1$ signal comes solely from the ionization electrons collected in the corresponding section of gas. However, the charge is small and requires significant electronic amplification using preamplifiers and amplifiers. This has the dual effect of worsening the resolution and increasing the noise.

On the other hand, a smaller amount of electrons is produced in the section of gas under the MicroMegas but each electron creates an avalanche in the amplification gap. As such the charge collected is large enough to require little, if any, electronic gain leading to less noise and better resolution.

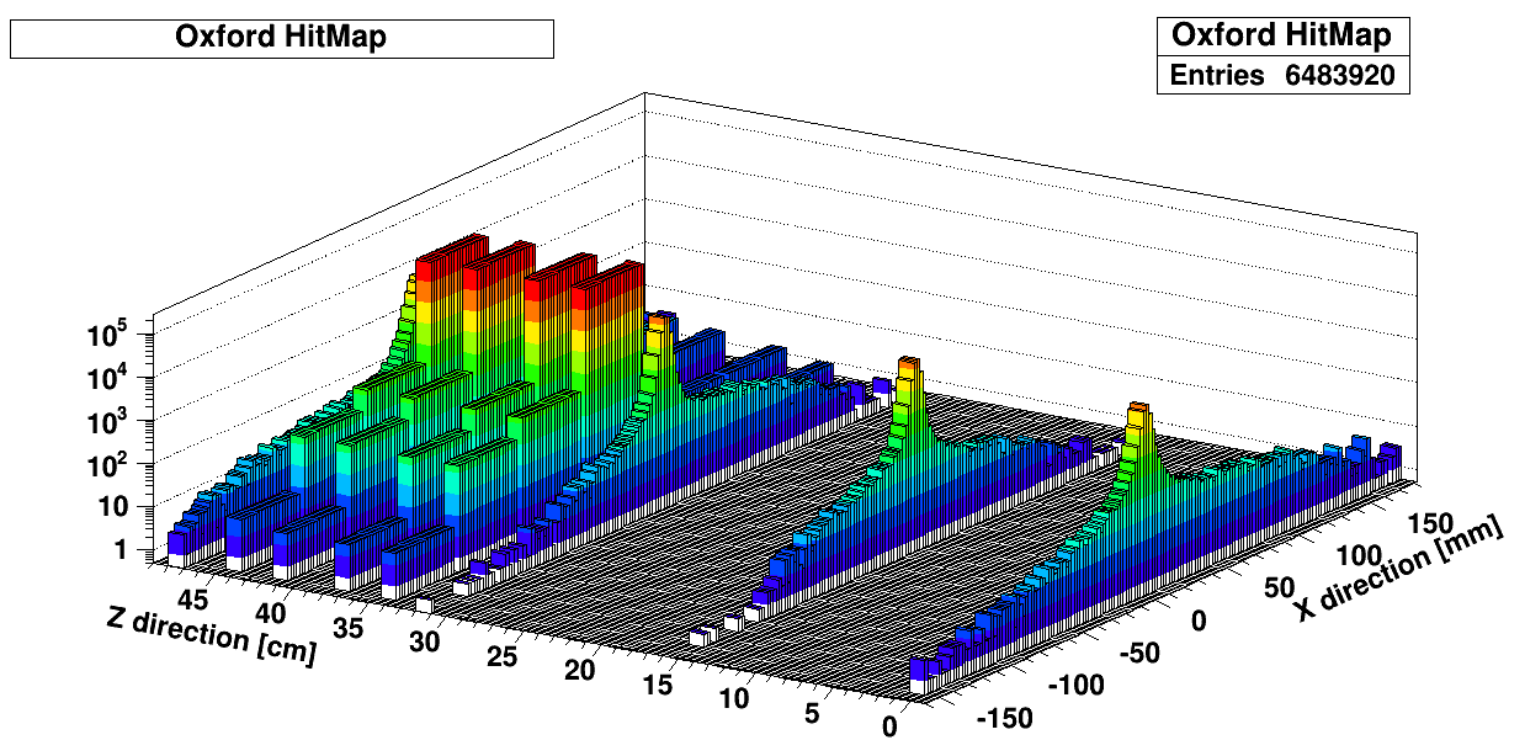

Figure 4. A map of the Oxford detector showing the 4 position-sensitive avalanche counters and the Micromegas pads. The peaks show that the beam is passing through the center of the detector highlighting predominantly column 4 (central) of the Micromegas anode. X-axis is along the wires (ACs). Positive direction corresponds to higher reaction angles and higher energy particles. Z-axis is along the beam. Positive direction is downstream. The position of the first $\mathrm{AC}$ is taken as the 0 of the axis.

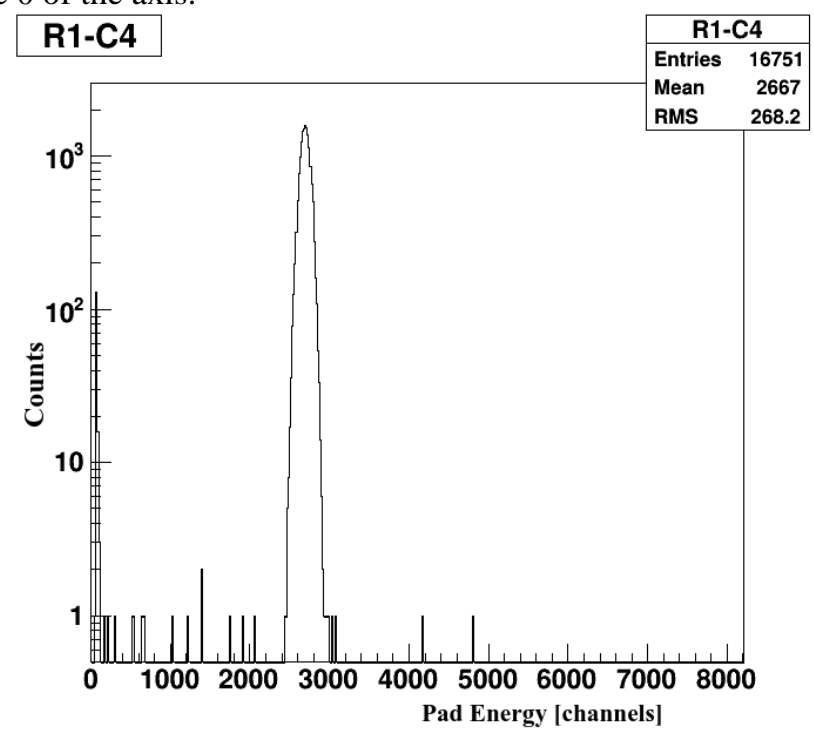

Figure 5. Energy spectrum for pad R1-C4 of the MicroMegas anode. 


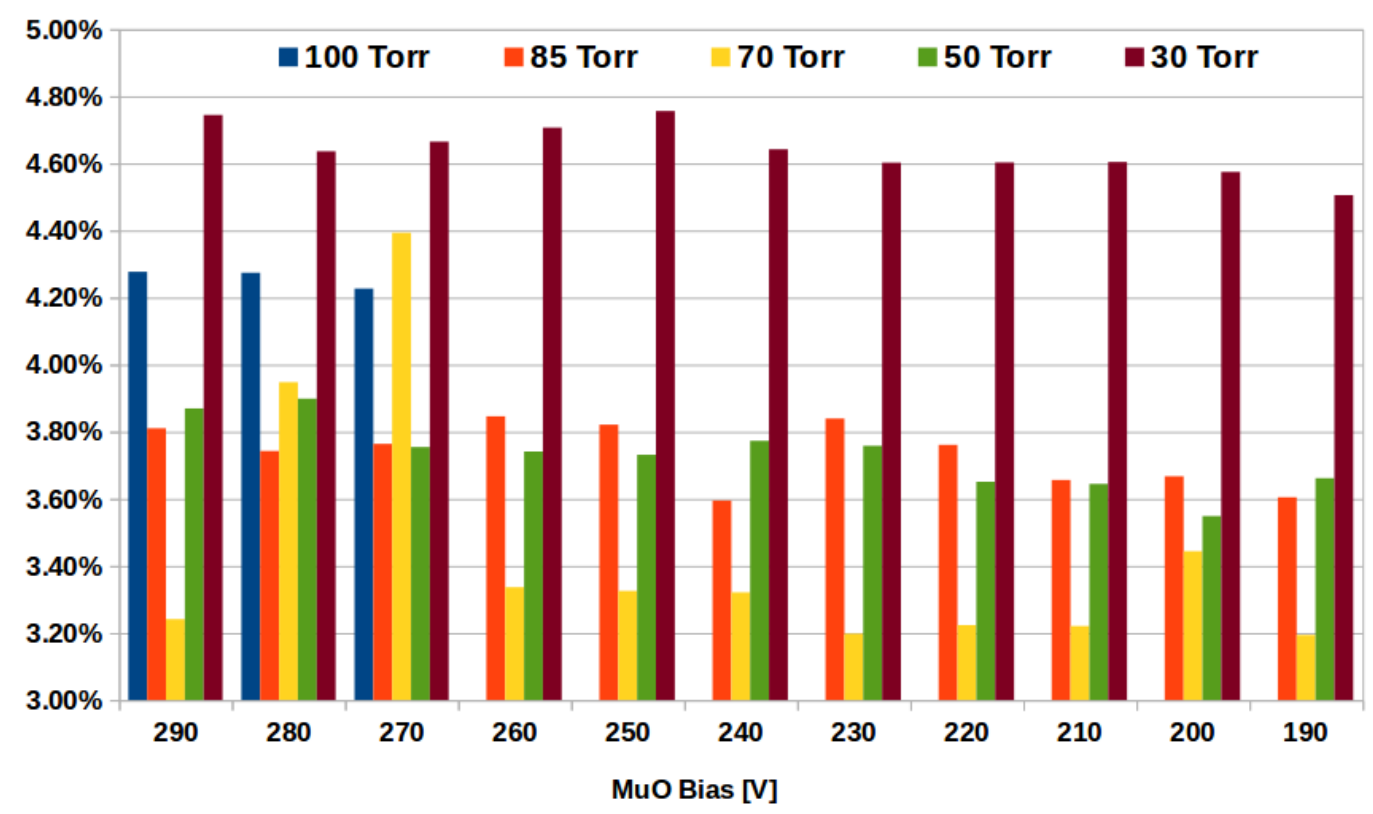

Figure 6. Plot showing the energy loss resolution for the Micromegas anode (averaged over the 28 pads) for different bias voltages of the Micromegas and different gas pressures when detecting ${ }^{22} \mathrm{Ne}+{ }^{197} \mathrm{Au}$ elastic scattering.

\section{Conclusions}

Using the MicroMegas to detect energy lost in the gas section of the Oxford detector led to energy resolutions 2-3 times better than with the ionization chamber. As such, the upgrade was considered successful and meets the requirements for particle identification in ANC studies so the modified detector is now being used in nuclear physics experiments. However, for a wider range of applications we would like to do further testing, with higher energy beams (20-40 $\mathrm{MeV} / \mathrm{u})$, heavier particles $\left({ }^{32} \mathrm{~S},{ }^{40} \mathrm{Ar} . ..\right)$, as well as different gas mixtures. In particular, the improved Oxford is also intended for future use in studies with re-accelerated unstable beams from the T-Rex upgrade of our facility [9]. 

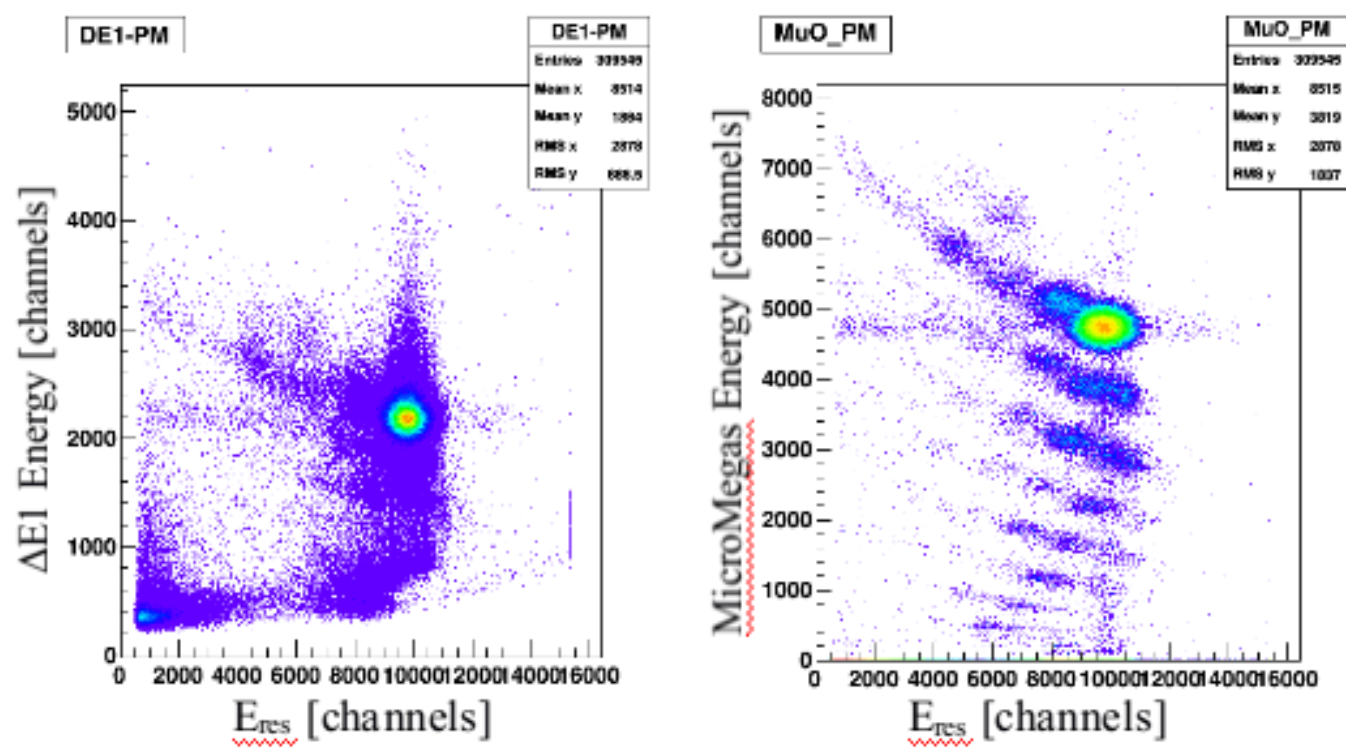

Figure 7. Spectrum on left shows energy detected by the ionization chamber, $\Delta \mathrm{E} 1$, versus $\mathrm{E}_{\mathrm{res}}$. Spectrum on right shows energy detected by the Micromegas versus same $\mathrm{E}_{\text {res }}$. The data in both cases is from ${ }^{22} \mathrm{Ne}$ on ${ }^{13} \mathrm{C}$ at 5 degrees.

\section{Acknowledgements}

This work has been supported in part by the US DOE under Grants DE-FG02-93ER40773 and DE-NA0001785.

\section{References}

[1] C.E. Rolfs, Rodney W.S., Cauldrons in Cosmos, University of Chicago Press, 1988

[2] H. Schatz, Nuclear Physics A 827 (2009) 26c - 33c

[3] A.M. Mukhamedzhanov et al, Nuclear Physics A631 (1998) 788c-792c

[4] D.M. Pringle et al, Nucl. Instr. and Meth. in Physics Research A245 (1986) 230-247

[5] D. Youngblood et al., Nucl. Instr. and Meth. in Physics Research A 361 (1995) 359

[6] E. Pollacco et al., Nucl. Instr. and Meth. in Physics Research A, 723, 102 (2013)

[7] Y. Giomataris et al., Nucl. Instr. and Meth. in Physics Research A 376 (1996) 29-35

[8] T. Al-Abdullah, Ph.D Thesis, Texas A\&M University (2007)

[9] R.E.Tribble et al.,Eur.Phys.J.Spec.Top.150(2007)225 